\title{
Filme hidrolipídico e elasticidade cutânea em crianças e adolescentes de Vassouras, Rio de Janeiro: estudo piloto quantitativo
}

\author{
Hydrolipidic fillm and skin elasticity in children and adolescents from Vassouras, Rio de Janeiro: \\ quantitative pilot study \\ Película hidrolipídica y elasticidad de la piel en niños y adolescentes de Vassouras, Rio de Janeiro: \\ estudio piloto cuantitativo
}

Recebido: 27/10/2021 | Revisado: 06/11/2021 | Aceito: 11/11/2021 | Publicado: 15/11/2021

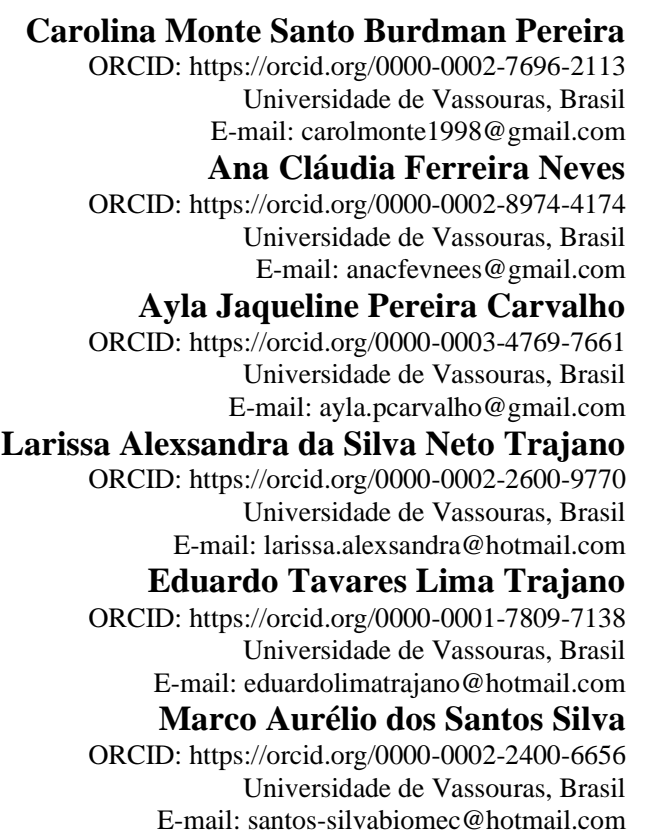

\section{Resumo}

Avaliar o FH, a TEWL e elasticidade cutânea em crianças e adolescentes por meio de variáveis como umidade e oleosidade da pele. Trata-se de um estudo piloto observacional, transversal e de natureza quantitativa realizado em escola privada de Vassouras em março de 2020, com 27 alunos de 11 a 15 anos. Os resultados demonstraram equilíbrio FH razoável na face, ruim na nuca e variação de razoável a ruim nos membros superiores e de bom a ruim nos inferiores. A elasticidade se mostrou não favorável na face, nuca e na maior parte dos membros superiores e inferiores. Os valores de TEWL variaram de acordo com a região e decorrem de mudanças na umidade e oleosidade, enquanto a elasticidade alterou devido à umidade.

Palavras-chave: Umidade; Anormalidades da pele; Estado de hidratação do organismo; Epiderme; Elasticidade.

\begin{abstract}
Evaluate the FH, the TEWL and skin elasticity in children and adolescents through variables such as skin humidity and oiliness. This is a pilot observational, cross-sectional study of a quantitative nature carried out in a private school in Vassouras, in March 2020, with 27 students between 11 and 15 years. The results demonstrate that a reasonable hydrolipidic balance was found on the face, bad on the neck and a range from reasonable to bad on the upper limbs and from good to bad on the lower limbs. Elasticity wasn't favorable on the face, neck and most of the upper and lower limbs. TEWL values varied according to the region and are due to changes in humidity and oiliness, while elasticity underwent changes due to humidity.
\end{abstract}

Keywords: Humidity; Skin abnormalities; Organism hydration status; Epidermis; Elasticity.

\section{Resumen}

Evaluar FH, TEWL y elasticidad de la piel en niños y adolescentes a través de variables como humedad y grasa de la piel. Se trata de un estudio piloto observacional y transversal de carácter cuantitativo realizado en un colegio privado 
de Vassouras en marzo de 2020, con 27 alumnos de entre 11 y 15 años. Los resultados mostraron un equilibrio de la HF razonable en la cara, deficiente en la nuca y una variación de regular a mala en las extremidades superiores y de buena a mala en las inferiores. La elasticidad fue desfavorable en la cara, la nuca y la mayoría de las extremidades superiores e inferiores. Los valores de TEWL variaron según la región y se deben a cambios en la humedad y oleosidad, mientras que la elasticidad cambió debido a la humedad.

Palabras clave: Humedad; Anomalías cutáneas; Estado de hidratación del organismo; Epidermis; Elasticidad.

\section{Introdução}

A pele tem função de proteção aos estímulos do ambiente e é dividida em hipoderme, derme e epiderme (Chang, Liu, Shao, \& Liao, 2017; Feingold, 2007; Arda, Göksügür, \& Tüzün, 2014; Sahle, Gebre-Mariam, Dobner, Wohlrab, \& Neubert, 2015). A hipoderme localiza-se abaixo da derme e é formada por tecido subcutâneo que contém gordura, exercendo função de preservar calor, proteger órgãos internos e permitir a movimentação da pele (Chang et al., 2017; Arda et al., 2014; Sahle et al., 2015; Losquadro, 2017). A derme é responsável pela espessura da pele e seu conteúdo fibroelástico é capaz de exercer proteção aos impactos externos (Chang et al., 2017; Sahle et al., 2015; Losquadro, 2017). É formada principalmente por colágeno, mas também abriga elastina, vasos sanguíneos, nervos, glândulas sebáceas e sudoríparas (Arda et al., 2014; Sahle et al., 2015; Losquadro, 2017). A elasticidade da pele é promovida principalmente pelas fibras elásticas e colágenas dispostas em seu interior, juntamente com a capacidade de renovação celular e a manutenção dos mecanismos envolvidos na hidratação da epiderme (MEDLIJ, 2015, Seção Desenvolvimento; Proksch et al., 2014).

A epiderme é dividida em camadas, sendo o estrato basal o mais profundo, seguido do estrato espinhoso, granuloso e córneo (EC), o mais superficial e responsável pela força da pele e sua resistência a danos (Chang et al., 2017; Arda et al., 2014; Sahle et al., 2015; Losquadro, 2017). O EC é formado por corneócitos envolvidos por matriz lipídica, composta principalmente por ceramidas, ácidos graxos livres e colesterol, formando uma camada hidrofóbica que realiza função de barreira da pele contra a perda de água transepidermal (TEWL) (Feingold, 2007; Sahle et al., 2015; Verdier-Sévrain \& Bonté, 2007). Além disso, as glândulas sebáceas liberam sebo na superfície cutânea, formado principalmente por triglicerídeos, conferindo a ela função de barreira protetora (Addor, 2016). A maior parte de água armazenada no EC está dentro dos corneócitos e uma epiderme saudável armazena em torno de $70 \%$ de água (Sahle et al., 2015).

O filme hidrolipídico (FH) é formado pelos lipídeos extracelulares e pela parte hidrópica, conferida por substâncias umectantes do EC, que promovem retenção intracelular de água. O equilíbrio do filme hidrolipídico (EFH) depende da composição bem estabelecida dos seus componentes. Somado a isso, a integridade do EC com equilíbrio no mecanismo de hidratação da pele, por meio da retenção da água intracelular, também adquire papel importante, funcionando como fator regulatório da TEWL (Gonçalves \& Campos, 2009; Medlij, Altoe-Adorno, Camilo, 2015). O equilíbrio é essencial para assegurar a hidratação da pele, a regulação da perda de água e eletrólitos, atuação contra o ressecamento e abrasão, manutenção da flexibilidade e formação da função de barreira de proteção que impede a penetração de agentes externos patogênicos (Gonçalves \& Campos, 2009; Pinto, Cavalcante \& Lima, 2020; Piccioni, García-Rodrigo, Pellegrini, Mazzocchetti \& Fargnoli, 2017; Medlij et al., 2015). A prevalência de substâncias lipídicas no espaço entre as células é um fator que regula a perda de água do corneócito (Medlij et al., 2015).

Alguns fatores influenciam diretamente na composição dos lipídeos do EC, como dermatite atópica (DA), psoríase e idade, causando um desequilíbrio do FH (DFH) (Sahle et al., 2015). Além da pele ficar seca e pruriginosa, a TEWL predispõe a entrada de substâncias alérgenas, irritantes e microrganismos através da epiderme, causando inflamação e aumentando as chances de infecção secundária (Sahle et al., 2015).

Apesar dos parâmetros relacionados à TEWL serem amplamente utilizados para definir disfunção da barreira cutânea, falta referências de trabalhos relacionados a padrões de valor médio de TEWL, umidade e oleosidade que indiquem suas manifestações clínicas. A definição desses padrões poderia ser útil para prevenção do número de manifestações dermatológicas 
e auxiliaria no tratamento adequado. O objetivo do presente foi avaliar o FH, TEWL e elasticidade cutânea em crianças e adolescentes do município de Vassouras, Rio de Janeiro, por meio das variáveis de umidade e oleosidade da pele.

\section{Metodologia}

Este estudo representa a análise de dados de um estudo piloto observacional, transversal e de natureza quantitativa realizado em escola particular do município de Vassouras, Rio de Janeiro, em março de 2020. Foram incluídos os estudantes com assinatura do Termo de Consentimento Livre e Esclarecido (TCLE) e Termo de Assentimento (TA) pelos responsáveis legais. O estudo foi aprovado pelo Comitê de Ética em Pesquisa (CEP) sob número 3.536.372.

Os dados contabilizam informações de 27 alunos com idade entre 11 e 15 anos. Este valor refere-se a 13,23\% da amostragem mínima ( $\mathrm{n}=204$ alunos) do estudo em andamento, calculada com 95\% de intervalo de confiança e 5\% de margem de erro.

A coleta de dados pode ser dividida em 3 fases:

I. Anamnese por meio de formulário para os responsáveis das crianças, a fim de identificar os dados dos participantes do estudo, condições médicas atuais e prévias e condições socioeconômicas.

II. Exame ectoscópico (sinais e/ou sintomas de dermatite e atopia) e anamnese dirigida em busca de possíveis lesões e apresentações clínicas no momento imediatamente anterior à medição.

III. Análise da umidade, oleosidade, equilíbrio e elasticidade da pele em 41 regiões do corpo de acordo com os locais mais comuns de manifestações de doenças dermatológicas com uso do instrumento de medição (Skyn Analiser Digital da Skin Up, modelo SKN1501, Brasil) que utiliza a bioimpedância da pele para demonstrar percentuais da umidade e oleosidade, além de verificar se a elasticidade está satisfatória ou não.

Os dados foram computados com o programa Excel e a análise estatística através do Software GraphPad Prism 8.2.1 for Windows. Os dados foram submetidos aos testes de normalidade de Komolgorov-Smirnov, Shapiro-Wilk e D'Agostinho e Pearson e aos testes T e Mann-Whitney para os dados paramétricos e não paramétricos, respectivamente. Para todos os dados foi considerado o valor de $\mathrm{p}<0,05$.

\section{Resultados}

\section{Análise da elasticidade da pele dos participantes}

A Tabela 1 evidencia que as peles da face dos participantes demonstraram elasticidade não favorável devido à redução da umidade ( $\mathrm{p}<0,0001)$. A elasticidade não favorável da nuca foi causada pela elevação da oleosidade. Embora o lado esquerdo da nuca tenha apresentado baixa umidade $(\mathrm{p}=0,0022)$, a oleosidade se apresentou elevada $(\mathrm{p}=0,0287)$, similar ao lado direito $(\mathrm{p}=0,0468)$. A elasticidade se apresentou não favorável devido à redução da umidade na fossa cubital direita $(\mathrm{p}<0,0001) \mathrm{e}$ esquerda ( $p<0,0001)$, entre o $1^{\circ}$ e $2^{\circ}$ dedos do pulso dorsal do lado direito $(p<0,0001)$, no pulso palmar do lado direito $(p<0,0001)$ e esquerdo ( $(\mathrm{p}<0,0001)$, entre o $4^{\circ}$ e $5^{\circ}$ dedos da mão palmar no lado direito ( $\left.<<0,0001\right)$ e esquerdo $(p<0,0001)$ e na região central da mão palmar do lado direito ( $\mathrm{p}<0,0001)$. Entretanto, as medidas realizadas entre o $4^{\circ}$ e $5^{\circ}$ dedos da mão dorsal do lado direito ( $p=0,0006)$ e esquerdo $(\mathrm{p}=0,0005)$, no pulso dorsal do lado esquerdo ( $\mathrm{p}<0,0001)$, entre o $1^{\circ}$ e $2^{\circ}$ dedos da mão dorsal do lado esquerdo $(\mathrm{p}<0,0001)$ e metacarpos do lado esquerdo $(\mathrm{p}=0,0001)$ revelaram que a elasticidade se apresentou não favorável devido à elevação da oleosidade. A elasticidade do pulso dorsal do lado direito se apresentou não favorável tanto pela redução da umidade ( $\mathrm{p}=0,0001)$ quanto pela elevação da oleosidade $(\mathrm{p}=0,0001)$. 
Tabela 1 - Elasticidade da face, nuca e dos membros superiores dos participantes de acordo com umidade e oleosidade.

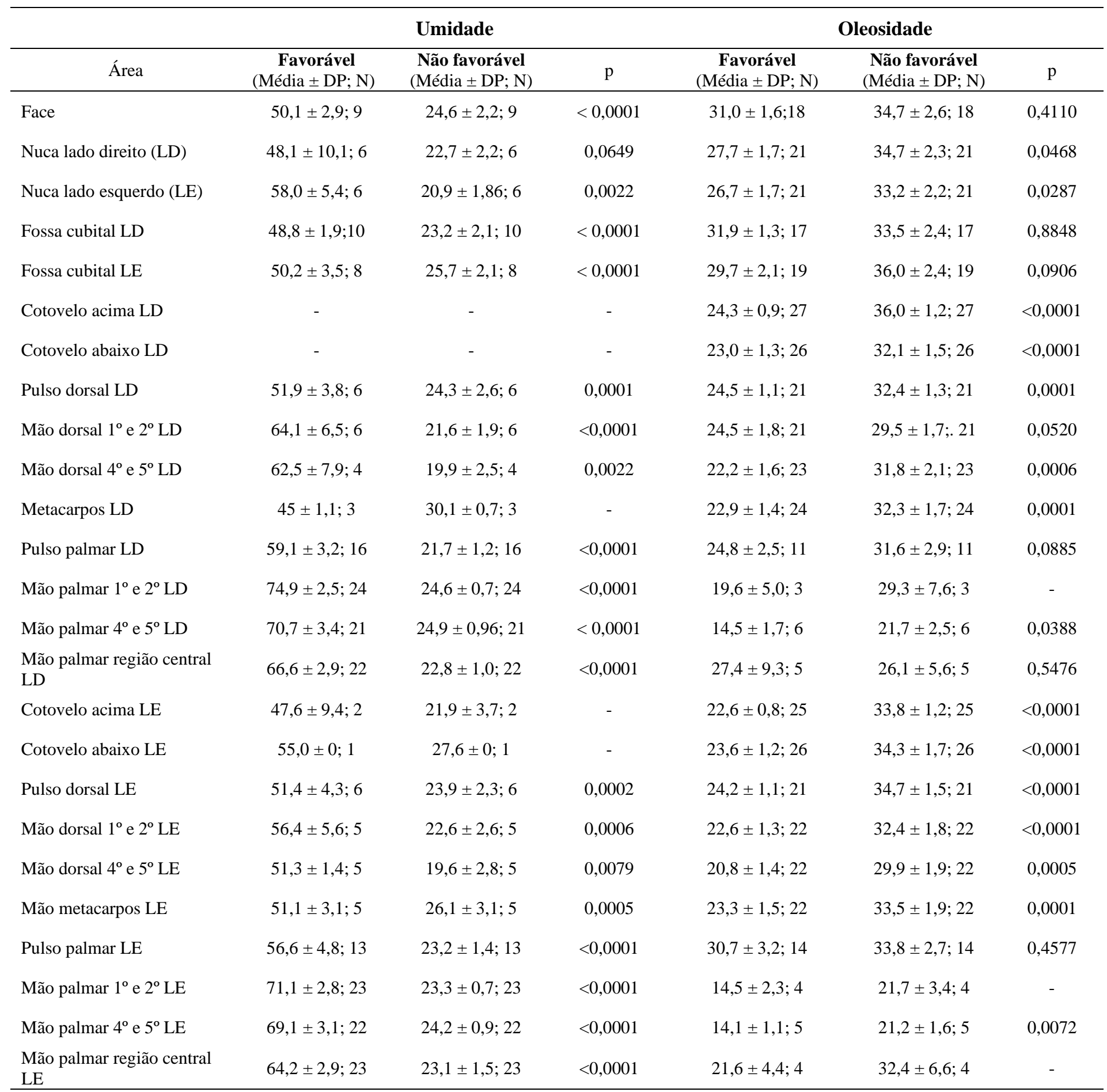

Legenda: $\mathrm{LD}$ = lado direito; $\mathrm{LE}=$ lado esquerdo; $\mathrm{N}$ = Número; $(-)$ = impossibilidade de análise por baixo $\mathrm{N}$ Fonte: Autores (2021). 
As análises das medições das regiões em MMII dos participantes demonstraram elasticidade não favorável entre o $1^{\circ} \mathrm{e}$ $2^{\circ}$ dedos do pé dorsal dos lados direito $(\mathrm{p}<0.0001)$ e esquerdo $(\mathrm{p}<0.0001)$, acima do $4^{\circ}$ dedo do pé dos lados direito $(\mathrm{p}<0.0001)$ e esquerdo ( $\mathrm{p}<0.0001)$, na região dorsal do pé abaixo do tornozelo dos lados direito ( $\mathrm{p}<0.0001)$ e esquerdo ( $\mathrm{p}<0.0001)$, na região central plantar do pé dos lados direito $(\mathrm{p}<0.0001)$ e esquerdo $(\mathrm{p}<0.0001)$ e no calcanhar na região plantar do pé do lado direito ( $\mathrm{p}<0.0001)$ devido à redução da umidade. Entre o $3^{\circ}$ e $4^{\circ}$ dedos do pé na região plantar dos lados direito $(\mathrm{p}<0.0001)$ e esquerdo $(\mathrm{p}<0.0001)$, a elasticidade se apresentou não favorável tanto pela redução da umidade, quanto pelo aumento da oleosidade (Tabela 2).

Tabela 2 - Elasticidade da pele dos membros inferiores dos participantes de acordo com umidade e oleosidade.

\begin{tabular}{|c|c|c|c|c|c|c|}
\hline \multirow[b]{2}{*}{ Área } & \multicolumn{3}{|c|}{ Umidade } & \multicolumn{3}{|c|}{ Oleosidade } \\
\hline & $\begin{array}{c}\text { Favorável } \\
(\text { Média } \pm \text { DP; N) }\end{array}$ & $\begin{array}{c}\text { Não favorável } \\
(\text { Média } \pm \text { DP; } N)\end{array}$ & $\mathrm{P}$ & $\begin{array}{c}\text { Favorável } \\
(\text { Média } \pm \text { DP; } N)\end{array}$ & $\begin{array}{c}\text { Não favorável } \\
(\text { Média } \pm \text { DP; N) }\end{array}$ & $\mathrm{p}$ \\
\hline Joelho lado direito (LD) & $54,5 \pm 3,1 ; 2$ & $17,4 \pm 1,0 ; 2$ & - & $30,0 \pm 1,6 ; 5$ & $39,2 \pm 4,4 ; 5$ & 0,0849 \\
\hline Joelho lado esquerdo (LE) & $56,0 \pm 2,9 ; 2$ & $17,9 \pm 0,9 ; 2$ & - & $29,9 \pm 1,6 ; 6$ & $44,8 \pm 2,4 ; 6$ & 0,0022 \\
\hline Pé dorsal $1^{\circ}$ e $2^{\circ} \mathrm{LD}$ & $56,3 \pm 2,3 ; 19$ & $21,9 \pm 1,0 ; 19$ & $<0,0001$ & $23,6 \pm 3,0 ; 8$ & $31,9 \pm 4,1 ; 8$ & 0,1290 \\
\hline Pé dorsal $4^{\circ}$ dedo $L D$ & $57,2 \pm 3,4 ; 12$ & $23,5 \pm 1,6 ; 12$ & $<0,0001$ & $23,3 \pm 2,0 ; 15$ & $30,8 \pm 2,0 ; 15$ & 0,0127 \\
\hline Pé dorsal abaixo tornozelo LD & $52,4 \pm 2,7 ; 10$ & $24,6 \pm 2,0 ; 10$ & $<0,0001$ & $23,7 \pm 1,6 ; 17$ & $33,9 \pm 9,6 ; 17$ & 0,0011 \\
\hline Pé plantar $1^{\circ}$ e $2^{\circ} \mathrm{LD}$ & $67,6 \pm 1,5 ; 26$ & $21,6 \pm 0,5$ & $<0,0001$ & $11,4 \pm 0 ; 1$ & $17,1 \pm 0 ; 1$ & - \\
\hline Pé plantar $3^{\circ}$ e $4^{\circ} \mathrm{LD}$ & $67,2 \pm 1,7 ; 22$ & $22,3 \pm 0,6 ; 22$ & $<0,0001$ & $14,1 \pm 1,5 ; 5$ & $24,3 \pm 2,2 ; 5$ & $<0,0001$ \\
\hline Pé plantar região central LD & $58,0 \pm 1,5 ; 22$ & $20,8 \pm 1,0 ; 22$ & $<0,0001$ & $19,9 \pm 4,3 ; 5$ & $27,3 \pm 5,5 ; 5$ & 0,3171 \\
\hline Pé plantar calcanhar LD & $65,3 \pm 1,7 ; 22$ & $21,4 \pm 0,5 ; 22$ & $<0,0001$ & $19 \pm 3,8 ; 5$ & $28,5 \pm 5,7 ; 5$ & 0,2045 \\
\hline Pé dorsal $1^{\circ}$ e $2^{\circ} \mathrm{LE}$ & $55,3 \pm 3,2 ; 15$ & $23,8 \pm 1,5 ; 15$ & $<0,0001$ & $23,5 \pm 2,4 ; 12$ & $30,0 \pm 2,2 ; 12$ & 0,0621 \\
\hline Pé dorsal $4^{\circ}$ dedo LE & $53,2 \pm 3,6 ; 8$ & $22,4 \pm 1,6 ; 8$ & $<0,0001$ & $23,7 \pm 1,7 ; 19$ & $32,4 \pm 2,1 ; 19$ & 0,0033 \\
\hline Pé dorsal abaixo tornozelo LE & $54,6 \pm 4,9 ; 7$ & $23,7 \pm 1,8 ; 7$ & $<0,0001$ & $24,9 \pm 1,5 ; 20$ & $32,9 \pm 1,8 ; 20$ & 0,0015 \\
\hline Pé plantar $1^{\circ}$ e $2^{\circ} \mathrm{LE}$ & $67,2 \pm 1,3 ; 27$ & $21,5 \pm 0,4 ; 27$ & $<0,000$ & - & - & - \\
\hline Pé plantar $3^{\circ}$ e $4^{\circ} \mathrm{LE}$ & $66,4 \pm 1,3 ; 22$ & $21,3 \pm 0,5 ; 22$ & $<0,0001$ & $11,2 \pm 0,3 ; 5$ & $16,7 \pm 0,4 ; 5$ & $<0,0001$ \\
\hline Pé plantar região central LE & $58,1 \pm 2,0 ; 20$ & $21,8 \pm 1,2 ; 20$ & $<0,0001$ & $21,5 \pm 3,5 ; 7$ & $27,6 \pm 3,0 ; 7$ & 0,2087 \\
\hline Pé plantar calcanhar LE & $66,4 \pm 1,9 ; 23$ & $21,2 \pm 0,6 ; 23$ & $<0,0001$ & $19,5 \pm 6,9 ; 4$ & $20,9 \pm 2,5 ; 4$ & - \\
\hline
\end{tabular}

Legenda: $\mathrm{LD}=$ lado direito; $\mathrm{LE}=$ lado esquerdo; $\mathrm{N}$ = Número; $(-)$ = impossibilidade de análise por baixo $\mathrm{N}$ Fonte: Autores (2021).

\section{Análise do EFH da pele dos participantes}

As medidas realizadas na face e na nuca (Tabela 3) revelaram que o equilíbrio entre umidade e oleosidade se apresentou razoável na face $(\mathrm{p}<0,0001)$. Embora o EFH do lado esquerdo da nuca tenha se apresentado razoável em alguns participantes $(\mathrm{p}=0,0001)$, a maioria apresentou equilíbrio ruim no lado esquerdo $(\mathrm{p}=0,0004)$ e direito $(\mathrm{p}=0,0006)$ por redução da umidade e elevação da oleosidade. 
Tabela 3 - Equilíbrio do filme hidrolipídico da pele da face, nunca e membros superiores dos participantes.

\begin{tabular}{|c|c|c|c|c|c|c|c|c|c|}
\hline \multirow{2}{*}{$\begin{array}{l}\text { Equilíbrio } \\
\text { Área }\end{array}$} & \multicolumn{3}{|c|}{ Amarelo } & \multicolumn{3}{|c|}{ Vermelho } & \multicolumn{3}{|c|}{ Verde } \\
\hline & $\begin{array}{c}\text { Umidade } \\
\text { (Média } \pm \text { DP } \\
\mathrm{N} \text { ) }\end{array}$ & $\begin{array}{c}\text { Oleosidade } \\
\text { (Média } \pm \text { DP } \\
\mathrm{N} \text { ) } \\
\end{array}$ & $\mathrm{p}$ & $\begin{array}{c}\text { Umidade } \\
\text { (Média } \pm \text { DP; } \\
\mathrm{N} \text { ) }\end{array}$ & $\begin{array}{c}\text { Oleosidade } \\
\text { (Média } \pm \text { DP } \\
\mathrm{N} \text { ) } \\
\end{array}$ & $\mathrm{p}$ & $\begin{array}{c}\text { Umidade } \\
\text { (Média } \pm \\
\text { DP; N) } \\
\end{array}$ & $\begin{array}{c}\text { Oleosidade } \\
\text { (Média } \pm \text { DP } \\
\mathrm{N} \text { ) } \\
\end{array}$ & $\mathrm{p}$ \\
\hline Face & - & - & - & $29,6 \pm 3,7 ; 10$ & $37,4 \pm 3,5 ; 10$ & 0,0753 & $\begin{array}{c}41,9 \pm 2,2 \\
17\end{array}$ & $27,7 \pm 2,2 ; 17$ & $<0,0001$ \\
\hline Nuca do LD & $68,8 \pm 0 ; 1$ & $22,0 \pm 0 ; 1$ & - & $22,4 \pm 1,6 ; 12$ & $33,6 \pm 2,3 ; 12$ & 0,0006 & $\begin{array}{c}41,0 \pm 2,7 \\
14\end{array}$ & $31,4 \pm 3,4 ; 14$ & 0,0136 \\
\hline Nuca do LE & $82,0 \pm 0 ; 1$ & $26,20 \pm 0 ; 1$ & - & $23,3 \pm 1,6 ; 15$ & $35,1 \pm 2,4 ; 15$ & 0,0004 & $\begin{array}{c}43,7 \pm 3,1 \\
11\end{array}$ & $\begin{array}{c}24,5 \pm 2,7 \\
11\end{array}$ & 0,0001 \\
\hline Fossa cubital LD & - & - & - & $27,8 \pm 1,8 ; 8$ & $37,9 \pm 2,8 ; 8$ & 0,0095 & $42,5 \pm 1, ; 19$ & $26,3 \pm 2,1 ; 19$ & $<0,0001$ \\
\hline Fossa cubital LE & $70,7 \pm 0 ; 1$ & $22,6 \pm 0 ; 1$ & - & $25,1 \pm 1,6 ; 11$ & $37,5 \pm 2,5 ; 11$ & 0,0004 & $\begin{array}{c}41,2 \pm 1,9 \\
15\end{array}$ & $30,3 \pm 2,8 ; 15$ & 0,0017 \\
\hline $\begin{array}{l}\text { Cotovelo acima } \\
\text { LD }\end{array}$ & - & - & - & $24,3 \pm 0,9 ; 27$ & $36,0 \pm 1,2 ; 27$ & $<0,0001$ & - & - & - \\
\hline $\begin{array}{l}\text { Cotovelo abaixo } \\
\text { LD }\end{array}$ & - & - & - & $21,8 \pm 1,0 ; 24$ & $32,7 \pm 1,6 ; 24$ & $<0,0001$ & $43,1 \pm 5,7 ; 3$ & $22,5 \pm 2,6 ; 33$ & - \\
\hline Pulso dorsal LD & - & - & - & $22,5 \pm 0,9 ; 18$ & $33,7 \pm 1,3 ; 18$ & $<0,0001$ & $46,7 \pm 3,6 ; 9$ & $24,3 \pm 1,7 ; 9$ & $<0,0001$ \\
\hline $\begin{array}{l}\text { Mão dorsal } 1^{\circ} \text { e } 2^{\circ} \\
\text { LD }\end{array}$ & $69,7 \pm 0 ; 1$ & $23,2 \pm 0 ; 1$ & - & $20,7 \pm 1,4 ; 16$ & $31,0 \pm 2,0 ; 16$ & 0,0002 & $49,8 \pm 5,8 ; 10$ & $23,0 \pm 1,2 ; 10$ & $<0,0001$ \\
\hline $\begin{array}{l}\text { Mão dorsal } 4^{\circ} \text { e } 5^{\circ} \\
\text { LD }\end{array}$ & $85,6 \pm 0 ; 1$ & $27,3 \pm ; 1$ & - & $20,8 \pm 1,4 ; 21$ & $31,2 \pm 2,1 ; 21$ & 0,0001 & $47,4 \pm 4,8 ; 5$ & $25,7 \pm 6,2 ; 5$ & 0,0317 \\
\hline Metacarpos LD & - & - & - & $21,1 \pm 1,2 ; 21$ & $31,4 \pm 1,7 ; 21$ & $<0,0001$ & $40,1 \pm 2,4 ; 6$ & $34,4 \pm 3,7 ; 6$ & 0,1645 \\
\hline Pulso palmar LD & $75,8 \pm 6,0 ; 4$ & $24,2 \pm 1,9 ; 4$ & - & $20,7 \pm 1,9 ; 8$ & $31,0 \pm 2,8 ; 8$ & 0,0084 & $50,0 \pm 2,5 ; 15$ & $23,4 \pm 2,3 ; 15$ & $<0,0001$ \\
\hline $\begin{array}{l}\text { Mão palmar } 1^{\circ} \mathrm{e} \\
2^{\circ} \mathrm{LD}\end{array}$ & $81,0 \pm 1,4 ; 18$ & $25,9 \pm 0,4 ; 18$ & $<0,0001$ & $19,6 \pm 5,1 ; 3$ & $29,3 \pm 7,6 ; 3$ & - & $56,5 \pm 2,5 ; 6$ & $20,6 \pm 2,0 ; 6$ & 0,0022 \\
\hline $\begin{array}{l}\text { Mão palmar } 4^{\circ} \mathrm{e} \\
5^{\circ} \mathrm{LD}\end{array}$ & $81,1 \pm 2,0 ; 13$ & $26,0 \pm 0,7 ; 13$ & $<0,0001$ & $14,5 \pm 1,7 ; 6$ & $21,7 \pm 2,5 ; 6$ & 0,0388 & $53,6 \pm 2,7 ; 8$ & $23,1 \pm 2,2 ; 8$ & 0,0002 \\
\hline $\begin{array}{l}\text { Mão palmar } \\
\text { região central LD }\end{array}$ & $79,2 \pm 2,7 ; 10$ & $25,3 \pm 0,8 ; 10$ & $<0,0001$ & $18,4 \pm 2,8 ; 4$ & $27,6 \pm 4,3 ; 4$ & - & $\begin{array}{l}56,7 \pm \\
1,5 ; 13\end{array}$ & $20,6 \pm 1,4 ; 13$ & $<0,0001$ \\
\hline $\begin{array}{l}\text { Cotovelo acima } \\
\text { LE }\end{array}$ & - & - & - & $22,6 \pm 0,8 ; 25$ & $33,8 \pm 1,2 ; 25$ & $<0,0001$ & $47,6 \pm 9,4 ; 2$ & $21,9 \pm 3,7 ; 2$ & - \\
\hline $\begin{array}{l}\text { Cotovelo abaixo } \\
\text { LE }\end{array}$ & - & - & - & $21,8 \pm 1,0 ; 22$ & $32,7 \pm 1,6 ; 22$ & $<0,0001$ & $38,1 \pm 4,3 ; 5$ & $40,0 \pm 5,7 ; 5$ & $>0,999$ \\
\hline Pulso dorsal LE & $68,0 \pm 0 ; 1$ & $21,7 \pm 0 ; 1$ & - & $23,1 \pm 0,9 ; 19$ & $34,5 \pm 1,3 ; 19$ & $<0,0001$ & $44,1 \pm 3,4 ; 7$ & $27,9 \pm 4,1 ; 7$ & 0,0105 \\
\hline $\begin{array}{l}\text { Mão dorsal } 1^{\circ} \text { e } 2^{\circ} \\
\text { LE }\end{array}$ & $77,0 \pm 0 ; 1$ & $24,6 \pm 0 ; 1$ & - & $21,4 \pm 1,2 ; 20$ & $32,0 \pm 1,8 ; 20$ & $<0,0001$ & $45,7 \pm 4,0 ; 6$ & $26,9 \pm 4,8 ; 6$ & 0,0135 \\
\hline $\begin{array}{l}\text { Mão dorsal } 4^{\circ} \text { e } 5^{\circ} \\
\text { LE }\end{array}$ & - & - & - & $19,5 \pm 1,2 ; 20$ & $29,2 \pm 1,8 ; 20$ & $<0,0001$ & $46,5 \pm 3,3 ; 7$ & $21,4 \pm 4,5 ; 7$ & 0,0041 \\
\hline $\begin{array}{l}\text { Mãometacarpos } \\
\text { LE }\end{array}$ & - & - & - & $22,1 \pm 1,3 ; 20$ & $33,1 \pm 1,9 ; 20$ & $<0,0001$ & $46,8 \pm 2,6 ; 7$ & $29,4 \pm 3,4 ; 7$ & 0,0065 \\
\hline Pulso palmar LE & $61,0 \pm 21,7 ; 3$ & $26,6 \pm 0,9 ; 3$ & - & $21,9 \pm 1,7 ; 7$ & $32,8 \pm 2,5 ; 7$ & 0,0032 & $48,7 \pm 3,0 ; 17$ & $21,4 \pm 2,7 ; 17$ & $<0,0001$ \\
\hline $\begin{array}{l}\text { Mão palmar } 1^{\circ} \mathrm{e} \\
2^{\circ} \mathrm{LE}\end{array}$ & $79,5 \pm 1,3 ; 15$ & $25,4 \pm 0,4 ; 15$ & $<0,0001$ & $14,5 \pm 2,3 ; 4$ & $21,7 \pm 3,4 ; 4$ & - & $55,4 \pm 3,0 ; 8$ & $19,3 \pm 0,9 ; 8$ & $<0,0001$ \\
\hline $\begin{array}{l}\text { Mão palmar } 4^{\circ} \mathrm{e} \\
5^{\circ} \mathrm{LE}\end{array}$ & $79,7 \pm 1,7 ; 13$ & $25,4 \pm 0,5 ; 13$ & $<0,0001$ & $14,1 \pm 1,1 ; 5$ & $21,2 \pm 1,6 ; 5$ & 0,0072 & $53,9 \pm 2,9 ; 9$ & $22,3 \pm 1,8 ; 9$ & $<0,0001$ \\
\hline $\begin{array}{l}\text { Mão palmar } \\
\text { região central LE }\end{array}$ & $78,8 \pm 1,7 ; 9$ & $25,2 \pm 0,5 ; 9$ & $<0,0001$ & $21,6 \pm 4,4 ; 4$ & $32,4 \pm 6,6 ; 4$ & - & $54,8 \pm 2,2 ; 14$ & $21,7 \pm 2,3 ; 14$ & $<0,0001$ \\
\hline
\end{tabular}

Amarelo = bom; Vermelho = rum; Verde = razoável $\mathrm{LD}=$ lado direito LE = lado esquerdo; $\mathrm{N}=$ Número; $(-)$ = impossibilidade de análise por baixo $\mathrm{N}$ Fonte: Autores (2021).

A Tabela 4 apresenta o EFH da pele dos MMSS dos participantes. O resultado revelou bom equilíbrio apenas entre o $1^{\circ}$ e $2^{\circ}$ dedos da mão palmar nos lados direito $(\mathrm{p}<0,0001)$ e esquerdo $(\mathrm{p}<0,0001)$, entre o $4^{\circ}$ e $5^{\circ}$ dedos da mão palmar nos lados 
direito ( $p<0,0001)$ e esquerdo ( $p<0,0001)$, na região central da mão palmar nos lados direito $(p<0,0001)$ e esquerdo ( $p<0,0001)$. A maioria dos participantes apresentaram áreas de pele dos MMSS com EFH razoável ou ruim. Foi demonstrado equilíbrio razoável, principalmente nas áreas da fossa cubital do lado direito ( $\mathrm{p}<0,0001)$, entre o $1^{\circ}$ e $2^{\circ}$ dedos da mão dorsal no lado direito $(p<0,0001)$, pulso palmar dos lados direito $(p<0,0001)$ e esquerdo $(p<0,0001)$. Enquanto, o EFH ruim da pele foi encontrado principalmente nas áreas da fossa cubital do lado esquerdo $(\mathrm{p}=0,0004)$, entre o $4^{\mathrm{o}}$ e $5^{\mathrm{o}}$ dedos na mão dorsal dos lados direito $(\mathrm{p}=0,0001)$ e esquerdo $(\mathrm{p}<0,0001)$, metacarpos dos lados direito $(\mathrm{p}<0,0001)$ e esquerdo $(\mathrm{p}<0,0001)$, pulso dorsal nos lados direito $(p<0,0001)$ e esquerdo ( $p<0,0001)$, entre o $1^{\circ}$ e $2^{\circ}$ dedos da mão dorsal no lado esquerdo ( $\left.p<0,0001\right)$ e abaixo do cotovelo no lado esquerdo $(\mathrm{p}<0,0001)$ devido a uma redução na umidade e elevação da oleosidade. No MMII, foi observado um bom EFH da pele da planta dos pés entre os $1^{\circ}$ e $2^{\circ}, 3^{\circ}$ e $4^{\circ}$ dedos nos lados direto $(p<0,0001)$ e esquerdo ( $\left.p<0,0001\right)$, bem como na parte plantar do pé na região calcânea nos lados direito $(p<0,0001)$ e esquerdo $(p<0,0001)$. O equilíbrio entre umidade e oleosidade se apresentou razoável principalmente na região dorsal do $1^{\circ}$ e $2^{\circ}$ dedos do pé dos lados direito $(p<0,0001)$ e esquerdo (p<0,0001), acima do $4^{\circ}$ dedo na região dorsal do pé nos lados direito $(\mathrm{p}<0,0001)$ e esquerdo $(\mathrm{p}<0,0001)$, na região dorsal do pé abaixo do tornozelo nos lados direito $(\mathrm{p}<0,0001)$ e esquerdo $(\mathrm{p}<0,0001)$, na região plantar central do pé nos lados direito $(\mathrm{p}<0,0001)$ e esquerdo $(\mathrm{p}<0,0001)$ devido a uma elevação da umidade e redução da oleosidade. O FH da pele se apresentou ruim principalmente no dorso do $4^{\circ}$ dedo do pé nos lados direito $(\mathrm{p}=0,0006)$ e esquerdo $(0,0003)$, além do dorso do pé abaixo do tornozelo no lado esquerdo ( $\mathrm{p}<0,0001$ ), por redução da umidade e elevação da oleosidade (Tabela 4). 
Tabela 4 - Equilíbrio do filme hidrolipídico da pele dos membros inferiores dos participantes.

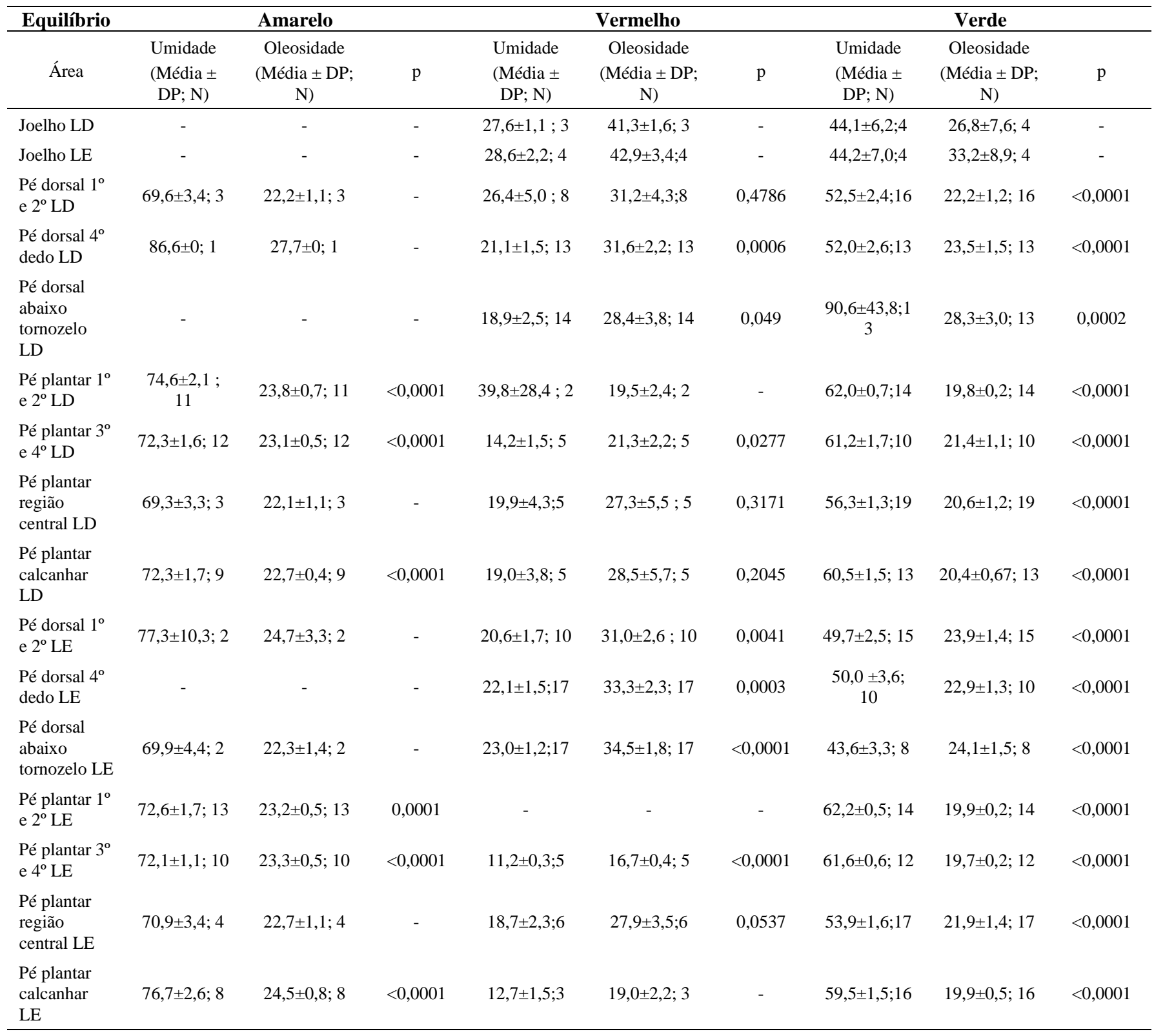

Amarelo = bom; Vermelho = ruim; Verde = razoável $\mathrm{LD}=$ lado direito $; \mathrm{LE}=$ lado esquerdo $; \mathrm{N}=$ Número; $(-)=$ impossibilidade de análise por baixo $\mathrm{N}$

Fonte: Autores (2021).

\section{Discussão}

O DFH é causado pela redução da presença de lipídios e/ou desidratação da pele, estresse, uso de produtos farmacêuticos e cosméticos, estilo de vida e alimentação, uso regular de nicotina e álcool (Gonçalves \& Campos, 2009; Piccioni et al., 2017; Butko, Tishakova, Lyapunov, Бутко, Тишакова, \& Ляпунов, 2019). O desequilíbrio tem como consequência o aumento da TEWL, promovendo esfoliação, descamação visível, opacidade e rachaduras (Beny, 2013; Proksch et al., 2014). Além disso, o FH não resiste às variações ambientais e agressões físicas, favorecendo o desenvolvimento de dermatites de contato cujo prurido mostra-se mais intenso à medida que a TEWL aumenta (Pinnagoda, Tupker, Coenraads \& Nater, 1990; Addor \& Aoki, 2010). Ademais, foi demonstrado uma redução progressiva nos valores de hidratação da pele verificada por meio da corneometria, de acordo com a intensidade do prurido, mesmo em áreas não lesionadas (Conti, Di Nardo \& Seidenari, 1996). 
Os valores de umidade, oleosidade, elasticidade e suas correlações determinadas por este trabalho indicam que, embora não visível, há disfunção da barreira cutânea na maior parte das medições realizadas, podendo indicar uma possível disfuncionalidade da pele ainda não diagnosticada (Addor \& Aoki, 2010).

O nível de hidratação do EC, assim como a TEWL, correlaciona-se com o grau de dano à barreira cutânea, construindo parâmetros biofísicos que permitem acompanhar os pacientes de maneira não invasiva e com maior grau de sensibilidade (Addor \& Aoki, 2010). Estudos comparando instrumentos como o Tewameter, o Evaporimeter e Cutometer, demonstraram que ruído aleatório, histerese do sensor e meio ambiente interferem na medição (Pinnagoda et al., 1990; Addor \& Aoki, 2010). Ainda não há na literatura dados sobre o instrumento utilizado neste estudo.

A temperatura do ar ambiente pode impactar na temperatura cutânea (Pinnagoda et al., 1990; Imhof, de Jesus, Xiao, Ciortea \& Berg, 2009; Levin \& Maibach, 2005; Barel \& Clarys, 1995). A pele apresenta uma estrutura variada de acordo com a região do corpo (Pinnagoda et al., 1990; Conti et al., 1996). Ademais, foi verificado diferença na aferição da TEWL com o equipamento Vaporemeter principalmente nas mãos, pés e axilas entre pacientes com e sem hiperidrose, em ambiente de temperatura controlada (Miotto et al., 2018). Há uma possível diferença entre sexos, com resultados maiores de TEWL em homens e de acordo com a idade, com redução da TEWL em idosos (Levin \& Maibach, 2005; Miotto et al., 2018). Ainda não há dados científicos da faixa etária estudada (entre 11 e 15 anos).

A distribuição das glândulas sebáceas e produção de sebo também varia de acordo com a região corporal (Conti et al., 1996). Há diferenças significativas relacionadas à sua produção quando comparado jovens e idosos, onde os jovens produzem mais principalmente na testa e parte superior das costas e menor na palma das mãos e sola dos pés (Conti et al., 1996; Sakuma \& Maibach, 2012). Em nosso estudo, foi verificado razoável EFH da face, bom equilíbrio na palma das mãos e em 3 pontos anatômicos dos 4 estudados na planta dos pés. Embora esses locais tenham maior concentração de glândulas sudoríparas, tanto a palma da mão quanto a planta do pé não possuem glândulas sebáceas como na face (Kashiwabara et al., 2016). Além disso, a face também apresenta maior exposição ao sol. Entretanto, o sebo parece não interferir na função de barreira da água e apresentar somente uma pequena participação na hidratação cutânea (Sakuma \& Maibach, 2012). Por isso, a umidade e a permeabilidade da barreira podem ser fatores importantes para determinar o DFH nesse caso. Ademais, há maior espessamento da pele na palma das mãos e planta dos pés quando comparadas com as demais partes do corpo, o que pode contribuir para um maior ressecamento (Kashiwabara et al., 2016). Entretanto, nossos achados de FH equilibrado indicam menor TEWL nessas regiões.

Regiões como o punho, pós-auricular e testa são mais expostas a fatores ambientais, com corneócitos menores, podendo resultar em maior permeabilidade quando comparados com braço, antebraço e abdome (Rougier, Lotte, Corcuff \& Maibach, 1988). O presente estudo demonstrou que mesmo com maior permeabilidade, a face apresentou-se com razoável EFH. Em contrapartida, apresentou-se com elasticidade baixa devido à redução da umidade. Embora o tamanho dos corneócitos das regiões supracitadas não mude, há grandes diferenças na TEWL, confirmando a importância da composição química do cimento intercelular nas propriedades de barreira do EC (Rougier et al., 1988). Dessa forma, este dado corrobora com o achado do nosso estudo, que apresenta variabilidade do valor de TEWL de acordo com a região e localidade do corpo (Conti et al., 1996). O presente estudo demonstrou que o pulso apresentou EFH razoável na região palmar e desequilíbrio na região dorsal nos lados direito e esquerdo.

A relação entre TEWL e a hidratação cutânea é amplamente debatida na literatura, uma vez que foi descrita uma relação inversa entre esses dois componentes em idosos saudáveis com pele clinicamente seca, não obstante outros autores não conseguiram encontrar correlação entre esses fatores em indivíduos hígidos. Por isso, é válido complementar que o ativo hidrolipídico da pele influenciando a barreira hídrica pode ser avaliado por parâmetros instrumentais que tenham valores quantitativos em condições patológicas e fisiológicas, de acordo com local, sexo e idade (Conti et al., 1996; Thune, Nilsen, Hanstad, Gustavsen \& Lövig, 1988). O aparelho utilizado no presente estudo realiza avaliação dos parâmetros através do toque 
de sua cabeça de medição na superfície da pele, utilizando a impedância bioelétrica para mensurar a umidade e oleosidade separadamente, bem como a sua relação, gerando dados referentes à elasticidade e FH.

Nos Guidelines for transepidermal waterloss (TEWL) measurement, um relatório publicado em 1990, mostrou-se que níveis basais de TEWL medidos pelo aparelho Evaporimeter EPI (Med) podem ser classificados da seguinte forma: palma > sola $>$ testa $=$ pele pós-auricular $=$ unha $=$ dorso da mão $>$ antebraço $=$ braço $=$ coxa $=$ tórax $=$ abdome $=$ costas, mostrando também que menores valores de TEWL encontram-se na região pré-tibial e panturrilha (Pinnagoda, et al., 1990; Conti et al., 1996). Ademais, foi demonstrado que a TEWL é aumentada principalmente na fronte e bochecha, relativamente aumentada na face interna do antebraço e reduzida na face interna da perna (Bernengo, Adhoute \& Mougin, 2015).

Foram feitas correlações entre a TEWL, hidratação e nível causal de sebo com os valores das regiões anatômicas do estudo e para todas as medições feitas em conjunto (93 indivíduos e 14 locais anatômicos), mas não mostraram relevância significativa (Miotto et al., 2018). Em nosso estudo, foi verificada relevância estatística entre a umidade e a elasticidade da pele, o que não ocorreu com a oleosidade. No entanto, os valores de oleosidade na face, fossa cubital do lado direito e esquerdo apresentaram-se maiores.

A idade e região anatômica são fatores importantes de influência para a viscoelasticidade da pele humana. Identificouse que a análise da viscoelasticidade da pele com o Cutometer sugeriu uma pele menos elástica no rosto do que no braço e nas costas (Ryu, Joo, Kim, Park \& Youn, 2008). Nossos achados mostraram elasticidade não favorável na face e na fossa cubital direita e esquerda devido a redução da oleosidade dessas regiões. Entretanto, a elasticidade do pulso dorsal do lado direito se apresentou não favorável pela redução da umidade e elevação da oleosidade. Como dito anteriormente, a hidratação está relacionada à capacidade de retenção de água pelo FH, mantendo a TEWL em níveis normais (Verdier-Sévrain \& Bonté, 2007). Dessa forma, um desequilíbrio destes componentes provoca aumento da TEWL e, por conseguinte, da elasticidade (Levin \& Maibach, 2005; Barel \& Clarys, 1995; Medlij et al., 2015; Proksch et al., 2014;).

\section{Conclusão}

Os dados deste estudo permitiram associação dos valores de umidade, oleosidade, elasticidade e EFH em diferentes partes do corpo. Os valores de TEWL variaram de acordo com a região e decorrem de mudanças na umidade e oleosidade, enquanto a elasticidade alterou devido à umidade.

Não foram encontrados estudos semelhantes na faixa etária analisada e/ou que levam em consideração as patologias cutâneas existentes. Por isso, como sugestão para futuros estudos científicos na área, recomenda-se maior diversidade nas faixas etárias, análise da história patológica pregressa e correlação dos dados das medições com os mesmos, assim como realizar um acompanhamento dos indivíduos do estudo a fim de identificar terapêuticas mais eficazes de acordo com os parâmetros de umidade e oleosidade.

\section{Agradecimentos}

Agradecimentos à Fundação Educacional Severino Sombra pela oportunidade de realização deste estudo científico.

\section{Referências}

Addor, F. A. S. Skin barrier in rosacea. (2016). An. Bras. Dermatol, 91(1), 59-63.

Addor, F. A. S., \& Aoki, V. (2010). Barreira cutânea na dermatite atópica. An. Bras. Dermatol., 85(2), 184-94

Arda, O., Göksügür, N., \& Tüzün, Y. (2014). Basic histological structure and functions of facial skin. Clinics in dermatology, 32(1), 3-13.

Barel, A., \& Clarys, P. (1995). Study of the Stratum corneum Barrier Function by Transepidermal Water Loss Measurements: Comparison between Two Commercial Instruments: Evaporimeter® and Tewameter®.Skin Pharmacol. Skin Pharmacol., 8(4), 186-95. 
Beny, M. (2013). Histologia e Fisiologia da Pele. Rev. Cosmetics e Toiletries Brasil, 25(2), 34-40.

Bernengo, J., Adhoute, H., \& Mougin, D. (2015). Measurement of the time of flight of photons into the skin: influence of site, age and gender, correlation with other skin parameters. Skin Res Technol, 21(1), 25-34.

Butko, Y., Tishakova, Т., Lyapunov, М., Бутко, Я., Тишакова, Т., \& Ляпунов, М. (2019). Development of Safe Dermotologic Preparation with Account of Natural Constituens of Skin. International Chemistry \& Biology Conference'19.

Chang, A. C., Liu, B. H., Shao, P. L., \& Liao, J. D. (2017). Structure-dependent behaviours of skin layers studied by atomic force microscopy. Journal of microscopy, 267(3), 265-271.

Conti, A., Di Nardo, A., \& Seidenari, S. (1996) No alteration of biophysical parameters in the skin of subjects with respiratory atopy. Dermatology, 192(4), 31720 .

Feingold, K. R. (2007). Thematic review series: skin lipids. The role of epidermal lipids in cutaneous permeability barrier homeostasis. Journal of lipid research, 48(12), 2531-2546.

Gonçalves, G. M. S., \& Campos, P. M. B. G. M. (2009). Aplicação de métodos de biofísica no estudo da eficácia de produtos dermocosméticos. Braz. J. Pharm. Sci, 45(1), 1-10.

Imhof, R., de Jesus, M., Xiao, P., Ciortea, L., \& Berg, E. (2009). Closed-Chamber Transepidermal Water Loss Measurement: Microclimate, Calibration And Performance. International Journal of Cosmetic Science, 31, 97-118.

Kashiwabara, T. B., Kashiwabara, Y. M. B., Rocha, L. L. V., Bacelar, L. F. F., França, P. L. V. L., \& Kashiwabara, L. M. (2016). Medicina Ambulatorial IV. Dejan Gráfica e Editora.

Levin, J., \& Maibach, H. (2005) The correlation between transepidermal water loss and percutaneous absorption: an overview. Journal Control Release, 103(2), 291-9.

Losquadro, W. D. (2017). Anatomy of the Skin and the Pathogenesis of Nonmelanoma Skin Cancer. Facial plastic surgery clinics of North America, 25(3), $283-289$.

Medlij, B. C., Altoe-Adorno, B. S., \& Camilo, K. F. B. (2015). Hidratação Cutânea: Aspectos Fundamentais na Manutenção e Reparação da Função Barreira da Pele. $15^{\circ}$ Congresso Nacional de Iniciação Científica, São Paulo, SP, Brasil.

Miotto, A., Honda, P. A. A., Bachichi, T. G., Holanda, C. S., Evangelista, N. E., Perfeito, J. A. J., \& Leão, L. E. V. (2018) Comparative study of transepidermal water loss in patients with and without hyperhidrosis by closed-chamber measurer in an air-conditioned environment. Einstein, $16(4)$, eAO4312.

Piccioni, A., García-Rodrigo, C., Pellegrini, C., Mazzocchetti, G., \& Fargnoli, M. (2017). Improving Skin Aging, Skin Hydration and Sensitive Skin with Four Specific Skin Care Products: Results from a Single-Centre, Observational, Prospective Study. Journal of Cosmetics, Dermatological Sciences and Applications, 7:48-56.

Pinnagoda, J., Tupker, R. A., Coenraads, P. J., \& Nater, J. P. (1990). Prediction of susceptibility to an irritant response by transepidermal water loss. Contact Dermatitis, 20(5), 341-6.

Pinto, E., Cavalcante, F., \& Lima, R. (2020). A fitoterapia no tratamento de pele: um estudo bibliográfico. Biodiversidade, 19(3), 189-195.

Proksch, E., Segger, D., Degwert, J., Schunck, M., Zague, V., \& Oesser, S. (2014). Oral supplementation of specific collagen peptides has beneficial effects on human skin physiology: a double-blind, placebo-controlled study. Skin pharmacology and physiology, 27(1), 47-55.

Rougier, A., Lotte, C., Corcuff, P., \& Maibach, H. (1988) Relationship between skin permeability and corneocyte size according to anatomic site, age, and sex in man. Journal of the Society of Cosmetic Chemists, 39, 15-26.

Ryu, H., Joo, Y., Kim, S., Park, K., \& Youn, S. (2008) Influence of age and regional differences on skin elasticity as measured by the Cutometer®. Skin Research and Technology, 14(3), 354-8.

Sahle, F. F., Gebre-Mariam, T., Dobner, B., Wohlrab, J., \& Neubert, R. H. (2015). Skin diseases associated with the depletion of stratum corneum lipids and stratum corneum lipid substitution therapy. Skin pharmacology and physiology, 28(1), 42-55.

Sakuma, T. H., \& Maibach, H. I. (2012) Oily Skin: An Overview. Skin Pharmacol Physiol, 25(5), 227-35.

Thune, P., Nilsen, T., Hanstad, I., Gustavsen, T., \& Lövig, D. H. (1988) The water barrier function of the skin in relation to the water content of stratum corneum, $\mathrm{pH}$ and skin lipids. The effect of alkaline soap and syndet on dry skin in elderly, non-atopic patients. Acta Derm Venereol, 68(4), $277-83$.

Verdier-Sévrain, S., \& Bonté, F. (2007). Skin hydration: a review on its molecular mechanisms. Journal of cosmetic dermatology, 6(2), 75-82. 